\title{
OSCILLATION OF SECOND ORDER NONLINEAR IMPULSIVE DIFFERENTIAL EQUATIONS WITH DEVIATING ARGUMENTS
}

\author{
E. Thandapani, R. SAKTHIVEL And E. CHANDRASEKARAN
}

Abstract. In this paper, we present some new sufficient conditions for the oscillations of all solutions of a second order retarded differential equations with impulses.These results extend the known results for the differential equations without impulses.An example is provided to illustrate our result.

Mathematics subject classification (2010): 34C10, 34A37.

Keywords and phrases: oscillation, second order, nonlinear, impulsive, differential equations.

\section{REFERENCES}

[1] L. Berezansky, E. Braverman, On oscillation of a second order impulsive delay differential equation, J. Math. Anal. Appl., 39 (2000), 217-225.

[2] E.M. BonotTo, L.P. Gimenses, M. Federson, Oscillation for a second-order neutral differential equation with impulses, Appl. Math. Comput., 215, 1 (2009), 1-15.

[3] J.-F. Cheng, Y.-M. ChU, Oscillation of second order neutral impulsive differential equations, J. Inequal. Appl., (2010), 29 pp.

[4] S.R. GRACE, Oscillation theorems for nonlinear differential equations of second order, J. Math. Anal. Appl., 171 (1992), 220-241.

[5] L.P. GIMENSES, M. FEDERSON, Oscillation by impulses for a second order delay differential eqution, Cad. Mat., 6 (2005), 181-191.

[6] K. Gopalsamy And B.G. Zhang, On delay differential equations with impulses, J. Math. Anal. Appl., 139 (1989), 110-122.

[7] I. GyoRI, G. LADAS, Oscillation theory of delay differential equations. With applications, Oxford University Press, New York, 1991.

[8] C. Guo, Z. XU, On the oscillation of second order linear impulsive differential equations, Differ. Equ. Appl., 2, 3 (2010), 319-330.

[9] Z. HE, W. GE, Oscillation of impulsive delay differential equations, Indian. J. Pure. Appl. Math., 31, 9 (2000), 1089-1101.

[10] Z. HAN, T. LI, S.Sun, W. CHEN,On the oscillation of second order neutral delay differential equations, Advances in Difference Equations, Volume (2010),Article ID 289340,8 pages.

[11] T. Kusano, H. Onose, Oscillation theorems for second order diffeential equations with retarded argument, Proc. Japan Acad., 50 (1974), 342-346.

[12] V. Lakshmikantham, D. Bainov, P.S. Simenov, Theory of Impulsive Differential Equations, Series in Modern Applied Mathematics, 6. World Scientific Publishing Co., Inc., Teaneck, NJ, 1989.

[13] W. XIU-LI, C. SI-YANG, H. JI, Oscillation of a class of second order nonlinear ODE with impulses, Appl. Math. Comput., 138 (2003), 181-188.

[14] Q. MEng, J. YAn, Bounded oscillation for second order non-linear neutral delay differential equations in critical and non-critical cases, Nonlinear Anal., 64, 7 (2006), 1543-1561.

[15] M. Peng, Oscillation caused by impulses, J. Math. Anal. Appl., 255 (2001), 163-176. 
[16] M. PENG, W. GE, Oscillation criteria for second order nonlinear differential equations with impulses, Comput. Math. Appl., 39 (2000), 217-255.

[17] M. PENG, R.P. AGARWAL, Oscillation theorems of second order nonlinear neutral delay differential equations under impulsive perturbations, Indian. J. Pure. Appl. Math, 33 (2002), 1017-1029.

[18] Ch.G. Philos, Oscillation theorems for linear differential equation of second order, Arch. Math. (Basel), 53, 5 (1989), 482-492.

[19] Y.V. Rogovchenko, Oscillation criteria for certain nonlinear differential equations, J. Math. Anal. Appl., 229 (1999), 399-416.

[20] Y.V. Rogovchenko, Oscillation of a second order nonlinear delay differential equations, Funkcial. Ekvac., 43 (2000), 1-29.

[21] F.S. SARYAL, A. ZAFER, Oscillation of second order nonlinear impulsive delay differential equations, Dyn. Contin. Discrete Impuls. Syst. Ser. A Math. Anal., 16, 2 (2009), 221-231.

[22] A.TIRYAKI, Oscillation criteria for a certain second order nonlinear differential equations with deviating arguments, Electron. J. Qual.Theory Differ. Equ., 61 (2009), 11 pp.

[23] Q. YANG, L. YANG, S. ZHU, Internval criteria for oscillation of second order nonlinear neutral differential equations, Comput. Math. Appl., 46 (2003), 903-918.

[24] J. YAN, Oscillation properties of a second order impulsive delay differential equation, Comput. Math. Appl., 47 (2004), 253-258.

[25] Y. Zhang, A. ZhaO, J. Yan, Oscillation criteria for impulsive delay differential equations, J. Math. Anal. Appl., 205 (1997), 461-470.

[26] A. ZHAO, J. YAN, Existence of positive solutions for delay differential equations with impulses, J. Math. Anal. Appl., 210 (1997), 667-678. 